\title{
A review of vagus nerve stimulation as a therapeutic intervention
}

This article was published in the following Dove Press journal: Journal of Inflammation Research

\author{
Rhaya L Johnson' \\ Christopher G Wilson ${ }^{1,2}$ \\ 'Lawrence D Longo MD Center for \\ Perinatal Biology, Department of \\ Basic Sciences, Loma Linda University, \\ Loma Linda, CA, USA; ${ }^{2}$ Department \\ of Pediatrics, Loma Linda University, \\ Loma Linda, CA, USA
}

\begin{abstract}
In this review, we provide an overview of the US Food and Drug Administration (FDA)approved clinical uses of vagus nerve stimulation (VNS) as well as information about the ongoing studies and preclinical research to expand the use of VNS to additional applications. VNS is currently FDA approved for therapeutic use in patients aged $>12$ years with drug-resistant epilepsy and depression. Recent studies of VNS in in vivo systems have shown that it has anti-inflammatory properties which has led to more preclinical research aimed at expanding VNS treatment across a wider range of inflammatory disorders. Although the signaling pathway and mechanism by which VNS affects inflammation remain unknown, VNS has shown promising results in treating chronic inflammatory disorders such as sepsis, lung injury, rheumatoid arthritis (RA), and diabetes. It is also being used to control pain in fibromyalgia and migraines. This new preclinical research shows that VNS bears the promise of being applied to a wider range of therapeutic applications. Keywords: vagus nerve stimulation, pediatrics, inflammation, peripheral nerve stimulation, autonomic circuits
\end{abstract}

\section{Introduction}

Vagus nerve stimulation (VNS) is US Food and Drug Administration (FDA) approved for use in the treatment of epilepsy and depression in patients aged $>12$ years and is currently being explored as treatment for a variety of other autoimmune and chronic inflammatory disorders, due to its demonstrated anti-inflammatory properties. ${ }^{1-6}$ In this review, we provide an overview of the evidence and diverse applications of VNS in clinical practice, clinical trials, and preclinical research. In addition, we provide a rationale for expanding the use of VNS to a wider range of patients across a wider range of diseases, including sepsis, lung injury, rheumatoid arthritis (RA), stroke, traumatic brain injury (TBI), obesity, diabetes, cardiovascular control, and pain management.

The vagus nerve provides an extensive afferent and efferent network of innervation for the viscera and plays a key role as an interface between higher central nervous system (CNS) circuits and the autonomic control circuitry of the brain stem. It is a mixed autonomic nerve originating at the medulla oblongata and projecting from the brain stem bilaterally along the neck (bundled with the carotid artery rostrally) and esophagus before branching diffusely to innervate the viscera. Most information about the anatomy of the vagus nerve and its projections has been discovered through tract tracing of the rat vagus and is generally assumed to be similar to humans. ${ }^{7}$ The complete innervation extent of the vagus remains incompletely known, but we have included a brief, simplistic overview of the primary branches and targets of the vagus.
Correspondence: Christopher G Wilson Lawrence D Longo MD Center for Perinatal Biology, Department of Basic Sciences, Loma Linda University, 2494 I Stewart St, Loma Linda, CA 92350, USA $\mathrm{Tel}+\mathrm{I} 9096515895$

Fax + I 9095584029

Email cgwilson@llu.edu 
The branches that extend off the cervical vagus innervate the bronchi, lungs, heart, and esophagus. ${ }^{7}$ The subdiaphragmatic vagus has five primary branches, including the dorsal and ventral gastric branches (innervating the stomach) as well as the dorsal and ventral celiac branches (innervating the proximal and descending colon). The hepatic branch divides into the hepatic branch proper (innervating the liver) and the gastroduodenal branch (innervating the duodenum and pancreas). ${ }^{1}$ The ventral trunk branches into the common hepatic, ventral gastric, and ventral celiac branches. The vagus consists of $\sim 80 \%$ sensory afferent and $20 \%$ motor efferent fibers. ${ }^{8}$ Further branching and tertiary targets for the vagus nerve are largely unknown. A fine wire electrode extends from the device and is typically wrapped around the left cervical vagus. Case reports suggest that the right vagus can be used in circumstances where approaching the left vagus is inadvisable. Since the right vagus innervates the sinoatrial node, stimulating on the right is best done with ECG monitoring.

The afferent projections of the vagus nerve are integrated at the level of the autonomic brain stem within the nucleus tractus solitarii (NTS) before projecting to other regions of the CNS. We have incomplete knowledge of how VNS modulates the CNS but the brain stem plays a critical role in integrating and gating signals between the CNS and peripheral organs (Figure 1). Descending efferents from these regions are responsible for driving cardiorespiratory and gastrointestinal autonomic tone as well as other autonomic functions. ${ }^{1,9}$ Stimulation of the vagus nerve provides a way to regulate the autonomic tone. Because the vagus nerve is easily accessible at the neck, it is a convenient access point for the implantation of stimulating or surface electrodes for chronic or acute stimulation.

\section{Current clinical uses of VNS} Treatment of epilepsy

Epilepsy affects $1 \%$ of the US population but costs $\$ 12$ billion US dollars to treat (figures from 2008). ${ }^{10} \mathrm{VNS}$ to treat epilepsy was first used in the early 1880 s by JL Corning, who believed that seizures were caused by changing cerebral blood flow. ${ }^{11}$ In 1988, the first chronic implantable stimulator was used to treat drug-resistant epilepsy. ${ }^{1}$ The stimulator was approved by the FDA in 1997 to treat partial onset seizures that were resistant to pharmacological control. ${ }^{1}$ The current Livanova $^{\odot}$ (formerly Cyberonics) implantable treatment device consists of a small battery-powered stimulator that requires battery removal and replacement approximately every 6 years. ${ }^{12} \mathrm{~A}$ fine wire electrode extends from the device

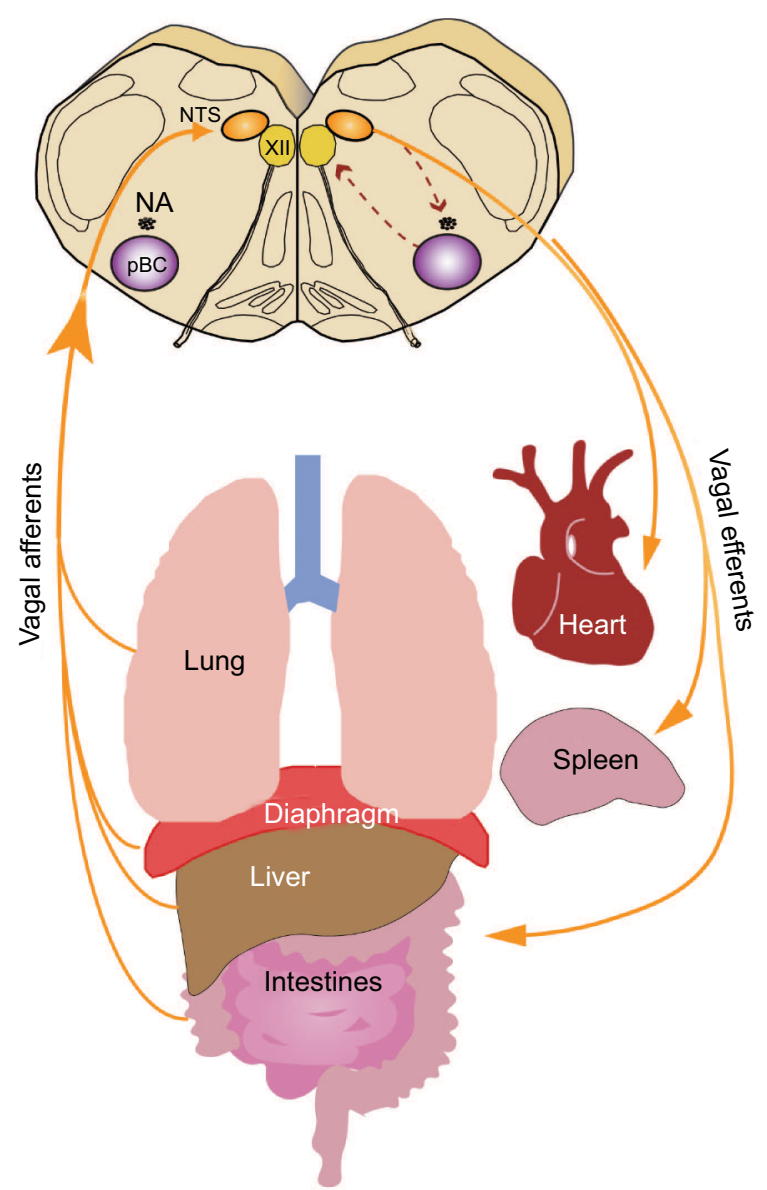

Figure I Overview of vagal circuitry linking the central and peripheral nervous system.

Notes: Visceral afferents converge on the NTS in the brain stem, the first point of integration between the peripheral autonomic nervous system and the central nervous system. Visceral afferents project from the dorsal motor nucleus of the vagus and are key to exerting autonomic control in the periphery.

Abbreviations: NA, nucleus ambiguus; NTS, nucleus tractus solitarii; $\mathrm{pBC}$, preBötzinger complex.

and is typically wrapped around the left cervical vagus. Case reports suggest that the right vagus can be used in circumstances where approaching the left vagus is inadvisable. Since the right vagus innervates the sinoatrial node, stimulating on the right is best done with ECG monitoring. ${ }^{13-15}$ The device can be turned on for 30-90 seconds to provide a brief stimulus to the vagus. ${ }^{16}$ Once the device is implanted, it is programmed by a physician using a microcomputer, but patients can alter the stimulus program as needed when they feel the onset of a seizure. ${ }^{17}$ Over 100,000 VNS devices have been implanted in patients worldwide (as of 2015). ${ }^{18}$ The most common side effects reported are dysphonia, hoarseness, and cough, all of which may be mitigated by changing stimulus parameters. ${ }^{19}$ Stimulation parameters vary widely, but typical treatment for epilepsy and depression uses a range of stimulation of 20-30 Hz, a pulse duration of up to 500 microseconds, and stimulation on-time of 30-90 seconds followed by off-time 
of 5 minutes, although stimulus intensity is usually decreased over time. ${ }^{16}$

Data gathered over the first decade of clinical VNS showed efficacy in patients who had pharmacoresistant seizures. Approximately $40 \%$ of patients using VNS showed a $50 \%$ reduction in seizures after $2-3$ years of treatment. ${ }^{20}$ The mechanisms by which VNS causes changes in neurochemistry and prevents epileptic seizures are not yet known, although some evidence suggests the vagus nerve plays a role in quenching kindling of seizures in regions susceptible to heightened excitability. These regions include the limbic system, thalamus, and thalamocortical projections. ${ }^{1} \mathrm{VNS}$ may also affect structures in the midbrain and hindbrain, which can contribute to seizure suppression, although the specific changes in these cortical circuits remain unknown. VNS also increases activity in the locus coeruleus and the raphe nuclei and moderates the downstream release of norepinephrine and serotonin, both of which have been shown to have antiepileptic effects. ${ }^{21}$ VNS success in treating refractory epilepsy with few side effects provides justification for its expansion to both additional conditions and wider populations. Figure 2 shows an overview of the CNS regions likely affected by VNS.

VNS may also be useful as a treatment for expecting mothers with treatment-resistant epilepsy. One study showed that women with epilepsy had a significantly higher risk of mortality during delivery when compared to women without epilepsy. ${ }^{22}$ The goal of current epilepsy treatment is to optimize seizure control and minimize in utero fetal exposure to antiepileptic drugs which, during the perinatal period, are associated with major congenital malformation, growth retardation, and neurocognitive developmental deficits. ${ }^{23}$ VNS has been used successfully as a treatment for medically refractory epilepsy in pregnant women, and physicians have concluded that VNS is a viable option for treatment during

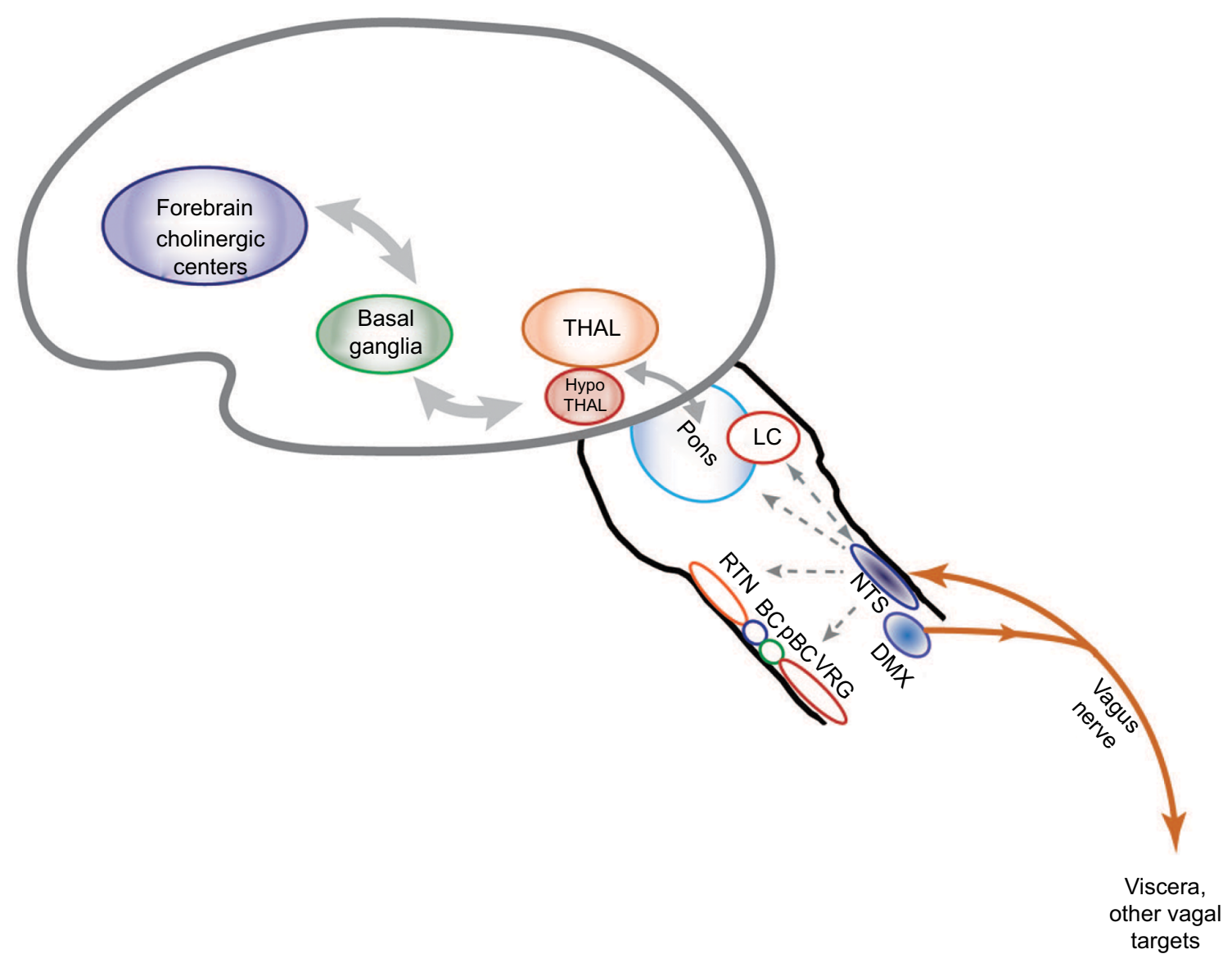

Figure 2 Putative pathways involved in vagus nerve stimulation.

Notes: Stimulation of the vagus activates ascending pathways that alter neural circuits in the brain stem, midbrain, and cortex. Regions that are impacted by vagus nerve stimulation based on past research are included in this diagram.

Abbreviations: NTS, nucleus tractus solitarii; DMX, dorsal motor nucleus of the vagus; LC, locus coeruleus; THAL, thalamus; HypoTHAL, hypothalamus; RTN, retrotrapezoid nucleus; $B C$, Bötzinger complex; $\mathrm{BC}$, preBötzinger complex; VRG, ventral respiratory group. 
pregnancy. ${ }^{24,25}$ As a non-pharmacological treatment, VNS seems to be beneficial for seizure control in the expecting mother, and there is no evidence of harm to the developing fetus. To date, no large clinical trials have been performed to assess whether VNS has a long-term effect on the developing fetus.

Epilepsy affects $0.5 \%-1 \%$ of the pediatric population ${ }^{19}$ or $\sim 470,000$ children. ${ }^{26}$ Chronic epileptic seizures can have a profound impact on children's long-term neurodevelopmental and social outcome, as well as a lasting impact upon their families. ${ }^{27}$ Finding an effective treatment can improve the children's quality of life since children with epilepsy often experience psychiatric and cognitive difficulties and have poor social outcomes as adults. ${ }^{27}$ Antiepileptic medications have high side effect profiles and can adversely affect the behavior in susceptible children. ${ }^{27}$ This means that even in children with more easily controlled epilepsy, there is a high risk of psychological and psychiatric disturbance. Children with benign rolandic epilepsy and absence epilepsy have exhibited more aggressive behavior, depression, and anxiety than children without epilepsy. ${ }^{28}$ Ongoing studies are focused on noninvasive methods to treat epilepsy in pediatric patients. This includes a study by the Chinese Academy of Chinese Medical Sciences testing a non-implant, less invasive transcutaneous auricular vagus nerve stimulator as an effective treatment of pediatric epilepsy. ${ }^{29}$ The study examines the change in frequency of seizures as well as heart rate variability, quality of life, and electroencephalogram recordings at 2, 4, and 6 months after the start of stimulation. ${ }^{29}$ In a retrospective cohort study by Elliott et al, ${ }^{19}$ the effects of VNS on 141 children were analyzed, $61 \%$ of whom were $<12$ years of age. They concluded that VNS was just as effective and relatively complication free in children aged $<12$ years as it was in older pediatric patients. This study also showed a significant decline in the frequency of seizures in these children, from an average of 10 per week down to three per week. In $41 \%$ of patients, there was a $75 \%$ reduction in the frequency of seizures. ${ }^{19}$ Side effects of VNS occurred in a small percentage of children and included hoarseness $(0.7 \%)$, cough $(0.7 \%)$, and minor arm pain $(0.5 \%) .{ }^{19}$ Hallböök et $\mathrm{al}^{30}$ found that pediatric responses to VNS were similar to those of adults. In $40 \%$ of children implanted with VNS stimulators, there was a $50 \%$ decrease in seizure frequency.$^{30} \mathrm{~A}$ retrospective study on 75 children with epilepsy showed that side effects such as hoarseness, cough, and drooling were reported in only 5.4\% of patients, in which all were reversible with adjustments to the stimulation parameters. ${ }^{31}$
Neonates could also benefit from a non-pharmacological approach to controlling epilepsy. Current pharmaceutical treatment options for epilepsy in neonates include phenobarbital and levetiracetam, although each can have detrimental side effects. Phenobarbital is the most widely used antiepileptic in neonates but can have respiratory and cognitive side effects. ${ }^{32}$ Levetiracetam has psychological side effects. ${ }^{33}$ Although VNS is only FDA approved in children aged $>12$ years, it has been used, along with antiepileptic medication, in children as young as 1 year of age. ${ }^{34} \mathrm{~A}$ study conducted by Fernandez et al on children aged $<3$ years showed that VNS was effective in children with medically intractable epilepsy. ${ }^{34}$ Their study showed that VNS led to decreased seizure frequency in $33 \%$ of patients, and status epilepticus was no longer a symptom after 1 year of treatment. In addition, normal MRIs were associated with decreased seizure incidence. $^{34}$

Children with developmental disabilities or autism are more likely to exhibit medically refractory epilepsy. One study estimated that between $5 \%$ and $38 \%$ of children with autism have epilepsy. ${ }^{35} \mathrm{~A}$ study by Kirchberger et $\mathrm{al}^{85}$ reported that VNS caused a $50 \%$ reduction in seizure frequency in $61 \%$ of patients with developmental delays. ${ }^{35}$ Levy et al also found that there was no statistically significant difference in seizure reduction benefits and quality of life improvements between patients with refractory epilepsy and autism and those without autism. ${ }^{35}$ Based on these results, VNS seems to be a safe and effective treatment for epilepsy in pediatric patients.

\section{Treatment of depression}

Chronic or severe depression affects up to $1.5 \%$ of the general population, ${ }^{8}$ and many of these patients obtain little relief from pharmaceutical treatment. In 2000, depression was estimated to cost $\$ 83.1$ billion in the United States. Of this cost, $\$ 26.1$ billion was in direct medical costs, $\$ 51.5$ billion in indirect workplace costs, and $\$ 5.4$ billion in suiciderelated mortality costs. ${ }^{36}$ Although VNS was not originally developed to treat depression, patients using VNS to treat epilepsy experienced mood improvement; thus, VNS was expanded to include the treatment of depression. The FDA approved VNS for the treatment of chronic or recurring depression in $2005 .{ }^{37} \mathrm{VNS}$ treatment has been approved for patients aged $\geq 18$ years who have experienced at least one major depressive episode and did not respond well to any of four different pharmaceutical antidepressant treatments. ${ }^{8} \mathrm{~A}$ major depressive episode is defined by the Diagnostic and 
Statistician Manual of Mental Disorders (DSM-IV) ${ }^{38}$ as having five out of the nine depressive symptoms, including depressed mood or lack of interest in normal day-to-day activities occurring almost daily for at least 2 weeks. ${ }^{39}$ The goal of treatment is to restore day-to-day function and prevent relapses and remission, as well as alleviate current symptoms, in which VNS has been proven effective in a wide range of patients. ${ }^{8}$ In a study conducted by Bajbouj et al, patients suffering from chronic "treatment refractory depression" received VNS, in which $53.1 \%$ of patients met the response criteria of a $50 \%$ reduction in the Hamilton Rating Scale for Depression (HRSD28) (the most commonly used symptom severity scale)..$^{37,40}$ In addition, $38.9 \%$ fulfilled the remission criteria with HRSD scores $<10 .{ }^{37}$

Depression is often difficult to treat because patients experiencing recurrent depressive episodes treated with conventional pharmaceuticals often experience relapses or do not experience full remission. A study conducted by Nahas et al showed patients with chronic or recurrent major depressive disorder receiving VNS may have beneficial long-term outcomes. ${ }^{41}$ In their study, $42 \%$ of their patients experienced a positive effect and $22 \%$ saw remission after 2 years. Both Bajbouj et al and Nahas et al use the same criteria for clinically significant remission, defined as the absence of clinically significant depressive symptoms. ${ }^{42}$ Results from neuroimaging studies suggest that the mood-enhancing benefits are caused by VNS stimulation altering medial and prefrontal cortical transmission (Figure 2). These regions comprise neurons that release neurotransmitters such as serotonin and norepinephrine which have both anticonvulsive and antidepressant effects. ${ }^{37}$ Although VNS will likely never be recommended as a "first-order" or sole treatment for depression, current clinical evidence shows success with its use as a supplemental treatment for chronic refractory depression.

Between $6 \%$ and $13 \%$ of pregnant women report symptoms of depression during and post pregnancy. ${ }^{43}$ The most commonly prescribed drug family for pregnant women with depression is selective serotonin reuptake inhibitors (SSRIs), although there are still unanswered questions about the safety of SSRI treatment for the fetus. ${ }^{43}$ Antidepressant use during pregnancy may lead to low birth weight and preterm delivery, since they can pass through the placenta. ${ }^{44}$ A case report by Husain et al showed that VNS was an effective treatment for depression during pregnancy and delivery with no adverse outcomes for the mother or fetus. ${ }^{45}$ A recent work in a rat model of VNS shows no significant effect of VNS on pups born to a dam with an implanted VNS stimulator. ${ }^{46}$ Preliminary research suggests that VNS can be a beneficial treatment for both mother and fetus, although more research is required for a clearer picture of the outcome.

Although depression also affects many adolescents, treatment options for pediatric patients are limited. Longitudinal studies on children with major depressive disorders have also shown that the relapse rate is $40 \%$ within 2 years and $70 \%$ within 5 years. ${ }^{47}$ Many children with major depression are treated using psychotherapy, but if their depressive symptoms persist, they are typically prescribed antidepressant medications in addition to therapy. ${ }^{48}$ As with pregnant mothers, the most common antidepressant medications prescribed in the pediatric population are SSRIs. ${ }^{48}$ As in adults treated with VNS, studies of pediatric patients with VNS implants to treat epilepsy have shown mood improvement. A study by Hallböök et $\mathrm{al}^{30}$ showed that, in children with epilepsy treated using VNS, not only were seizures reduced, but behavior and mood improved while depressive symptoms decreased. Twelve of the 15 children examined had improvement in their quality of life. ${ }^{30}$ Further studies are needed to examine the impact of VNS on pediatric depression, but the preliminary data show that it remains a promising treatment option and may provide long-term benefit for children with depression.

\section{Potential uses and mechanisms of VNS}

An exciting new application of VNS is as an anti-inflammatory treatment. Inflammation is implicated in many chronic diseases including cardiovascular disease, arthritis, and Alzheimer's disease. Preliminary preclinical evidence suggests that VNS may attenuate the inflammatory response through activation of the cholinergic anti-inflammatory pathway (CAP) - a long loop from the vagus afferents, through the autonomic brain stem and forebrain cortical structures, and then back through the descending vagus efferents (Figure 2). The CAP upregulates HMGB1, which may regulate cytokine expression, leading to anti-inflammatory effects. In recent years, Tracey et al have devoted significant efforts to quantifying the role that VNS plays as an anti-inflammatory regulator primarily through altered regulation of acetylcholine..$^{2-6}$ These findings provide strong evidence that stimulation of the vagus nerve plays a key role in peripheral cholinergic release and its putative role in suppressing inflammation. The CAP also affects the levels of acetylcholine through nicotinic acetylcholine receptors (nAChRs). ${ }^{2}$ Several recently completed and ongoing studies are focused on the effects of VNS on inflammatory disorders such as RA, Crohn's disease, irritable bowel syndrome, and fibromyalgia. Other studies are focused on how VNS affects brain trauma and stroke. Because these 
are ongoing studies, the efficacy of VNS treatment for these disorders is currently unknown. Many of the inflammatory disorders that VNS may potentially treat also affect the pediatric and neonatal population. Since VNS has been shown to be effective for adult and pediatric populations for epilepsy and depression, it stands to reason that VNS treatment may be beneficial to younger patients for a variety of disorders; however, there is limited data on pediatric applications. We summarize the results of studies related to these disorders below; however, these preliminary data provide a strong rationale for expanding research on the applications of VNS as an anti-inflammatory treatment across a range of different inflammatory diseases.

\section{Sepsis}

Sepsis is a multibillion dollar health care burden typically due to systemic bacterial infection and chronic activation of the pro-inflammatory cytokine cascade. Sepsis is estimated to cost $\$ 22,000$ per patient and affects up to 18 million individuals each year. ${ }^{49}$ Kessler et al used vagotomized mice to show that lack of vagus input to the CNS can lead to adverse outcomes in a murine model of colon ascendens stent peritonitis. ${ }^{50}$ In an ex vivo culture of Kupffer cells, tumor necrosis factor $\alpha$ (TNF $\alpha$ ) levels were decreased in vagotomized mice compared to controls, even when stimulated with lipopolysaccharide (LPS) ${ }^{50}$ Huang et al showed that VNS helped to attenuate inflammation by restoring the balance between parasympathetic and sympathetic tone and thus arresting the progression to sepsis. ${ }^{51}$ These investigators used an intravenous LPS injection model of inflammation to induce sepsis and found that in addition to a decrease in ACh release, heart rate variability was decreased back to baseline levels in the LPS + VNS treatment group, compared to the elevated levels in the LPS-only group. ${ }^{51}$ In a similar model of LPS-induced endotoxemia, Borovikova et al used VNS and found decreased mortality that they attributed to vagally induced release of acetylcholine. ${ }^{3}$

Limiting inflammation in pediatric patients without the use of pharmaceuticals is important because neonates, particularly preterm infants, are more susceptible to developing sepsis due to their underdeveloped immune systems and susceptibility to perinatal infection (chorioamnionitis, etc). Since VNS seems to regulate inflammation by modulating the cytokine cascade, our laboratory is looking at the effect of VNS on the early pro-inflammatory cytokines, interleukin-6 (IL-6), TNF $\alpha$, and IL-1 $\beta$, in respiratory control regions of the brain stem. We looked at the NTS and hypoglossal motor nucleus (XII), which are regions of critical importance for the control of breathing and implicated in breathing problems in neonatal rats as a model for preterm infants. In the present study, we show that VNS reduces the expression of IL- 6 and TNF $\alpha$ in response to a brief (30 minute) bout of high-frequency VNS stimuli. ${ }^{52}$ Our hope for this preclinical translational work is that it will eventually lead to minimally invasive VNS treatment to provide early intervention and reduce the likelihood of sepsis in preterm infants.

Two major concerns in extending VNS to neonatal practice are the length of time that VNS takes to be effective and the invasive nature of the implantation procedure. Although VNS as currently used for epilepsy and depression can take months to show dramatic effect, short-term stimulation has been used to reduce inflammation quickly, and our experiments have shown that a single bout of high-frequency stimulation (30 minutes) can be effective as an anti-inflammatory treatment. ${ }^{52}$ In addition, transcutaneous stimulation has been used to treat depression and headaches showing efficacy for even short-term applications using surface electrodes. ${ }^{29,53}$ A study by He et al uses transcutaneous stimulation at the cervical or auricular vagus to effectively treat epilepsy. ${ }^{29}$ Further research will be needed to determine if high-frequency stimulation can be paired with transcutaneous stimulation to be an effective treatment for neonatal inflammatory disorders. Preliminary work performed by the Wilson laboratory and others suggests that this may be possible and useful for implementation of VNS treatment in neonates. ${ }^{52,54,55}$

\section{Pain management}

The applications of VNS also extend to widespread inflammatory disorders associated with chronic or intermittent bouts of pain such as fibromyalgia and migraines. Lange et al conducted a Phase I/II clinical trial to assess VNS as an adjunct treatment for patients with fibromyalgia due to its effects on serotonergic and noradrenergic neural circuits both of which are implicated in pain sensation. ${ }^{56}$ Their theory was based on results from patients with depression treated with VNS who reported decreased sensation of pain. ${ }^{57}$ Lange et al's study included 12 women with fibromyalgia and used the same stimulus parameters as for treatment of epilepsy. After 11 months, 7 of the patients had the minimum clinical difference (MCID+) in their pain symptoms for VNS to be considered effective. ${ }^{56}$

Another chronic pain ailment that VNS shows promise in treating is migraine headaches. In a study conducted by Barbanti et al, 50 patients with migraine were given VNS treatment applied externally at the neck in two 120-second intervals with 3 minutes in-between. Of those patients, $56 \%$ 
reported pain relief at 1 hour and 64\% reported pain relief at 2 hours. ${ }^{53}$ Silberstein et al performed the ACT1 study (NCT01792817), a clinical trial to use a noninvasive VNS treatment at the neck to treat cluster headaches. ${ }^{58}$ Their findings suggest that noninvasive VNS can be successfully used to treat episodic cluster headaches. ${ }^{58}$ Although these results need further research and larger multicenter randomized trials, they present hopeful evidence that VNS can be used to control fibromyalgia and migraines.

\section{Obesity}

Although VNS would not likely be recommended as a firstline defense against obesity, research on the effects of VNS on diet and weight have been performed to evaluate VNS for its use as an adjunct treatment in controlling obesity. Finding alternative treatments to obesity is especially important considering $69 \%$ of adults and $20 \%$ of adolescents in the United States are overweight or obese. ${ }^{59}$ Burneo et al found that in patients implanted with VNS to control epilepsy, $62 \%$ experienced significant weight loss. ${ }^{60}$ Bodenlos et al ${ }^{61}$ conducted a study looking at the association between VNS and food cravings in depressed adults and found that left cervical VNS resulted in attenuated food craving. ${ }^{61}$ Recent work by Val-Laillet et al has shown that bilateral constant current stimulation of the vagus nerve led to lower food intake and sweet cravings in obese minipigs. ${ }^{62}$ This study did not suggest that VNS actually caused weight loss but rather that VNS helped prevent excess weight gain. The implications of these studies are compelling, but the mechanism by which VNS influences weight loss is still unknown. Some hypotheses include changes in metabolism, decreases in fat stores, or changes in satiety signaling. ${ }^{61}$ Another potential proposed mechanism for the effect of VNS on weight is reduced intestinal caloric absorption, which can be hypothesized based on the findings that vagal tone can alter peptides that can change gut motility and absorption. ${ }^{63}$ These preliminary results encourage more extensive research into VNS, and the impact of autonomic control modulation, and hypothalamic signaling and its interactions with the enteric nervous system.

A study performed by Ikramuddin et al showed the effect of VNS on morbid obesity. Since bariatric surgery presents some major risks, investigators are looking for alternative, less-invasive methods of controlling obesity. Their trial showed that weight loss was higher by a statistically significant margin in patients who underwent VNS when compared to sham patients. ${ }^{64}$ More research is needed, but implantation of VNS devices may be an attractive option for controlling weight and managing obesity for patients who have not had success with more traditional methods of weight control.

\section{Cardiovascular disease}

VNS must alter cardiovascular control due to the convergence of inputs in the autonomic control centers of the brain stem, but for how long and to what extent is unknown. The descending cardiac branch of the vagus is key for normal cardiac function. ${ }^{65}$ Atherosclerosis, which often predisposes one to coronary heart disease, is believed to be due to low-grade systemic inflammation. ${ }^{66}$ Since there is growing evidence that VNS is anti-inflammatory, it may provide another avenue for treating cardiac dysfunction and atherosclerosis. In the 2007 CARDIA study, Sloan et al showed that there was an inverse relationship between inflammatory markers and vagus nerve activity, measured by heart rate variability, suggesting that VNS is key to anti-inflammatory tone. They also suggest that high levels of pro-inflammatory markers such as IL-6 and $\mathrm{C}$-reactive protein may indicate a predisposition to coronary artery disease.$^{67} \mathrm{VNS}$ may also provide a therapeutic application for preventing heart failure. Zhang et al used a canine model to show that chronic VNS helps to regulate heart rate and improves heart function in a high-rate ventricular pacing model. ${ }^{68}$ In a rat ischemia/reperfusion (I/R) model, Zhao et al showed that VNS improved cardiac function and reduced infarct size. ${ }^{69}$ Their results also showed that VNS reduced mesenteric artery pathology and vasodilation caused by the I/R model and that lower levels of TNF $\alpha$ and IL-1 $\beta$ were found in serum. This is likely due to VNS effects on acetylcholine release and systemic levels and upregulation of M3AChR/a7nAChr expression ${ }^{69}$ which have been implicated in inflammatory modulation (see "Potential uses and mechanisms of VNS" section), but the precise mechanism is still unknown and needs further work. Chapleau et al used a high salt, spontaneously hypertensive rat model to show that right VNS prevented aortic stiffening and slowed the progression of endothelial dysfunction. ${ }^{70}$ In addition, they saw significantly higher serum IL-6 levels in VNS rats, which may indicate that VNS modulates inflammatory function in this severe hypertension model. ${ }^{70}$ Based on these studies, there is a relationship between cardiovascular disease, inflammation, and vagal activity that may be altered by VNS.

\section{Lung injury}

VNS is being considered as a treatment for ventilator-induced lung injury (VILI) caused by pressure-induced damage to lung alveoli. Inflammation has been shown to increase the 
likelihood of VILI, which is often the result of severe lung infection. Other respiratory disorders such as acute lung injury and acute respiratory distress syndrome, both of which can be complicated by sepsis, can also result in pronounced pulmonary inflammation. ${ }^{71-73}$ Experiments by dos Santos et al showed that the vagus nerve plays an important role in pulmonary inflammation. Interruption of the CAP by vagotomy leads to worsening VILI. ${ }^{71}$ Vagotomized animals with mechanical ventilation had increased alveolar damage and levels of IL-6 and hemorrhage compared to control animals. ${ }^{71}$ Later experiments showed that VNS, both electrical and pharmacological, attenuated the lung injury in a "two-hit" model of VILI (I/R injury followed by high tidal volume ventilation, which can be further injurious to the lung) by decreasing the pro-inflammatory and pro-apoptotic responses. $^{71}$

VNS may also be useful in treating gut and lung injuries together. In a study of lung injury caused by hemorrhagic shock, Reys et al showed that VNS prevents intestinal barrier failure and protects against lung injury. ${ }^{74}$ In addition, pharmacologic blockade of nicotinic cholinergic receptors in an in vitro culture model of pulmonary endothelial cells suggests that VNS acts through the CAP to prevent lung injury and gut-barrier breakdown. ${ }^{73}$ A study by Levy et al also showed that VNS alleviated lung injury caused by trauma hemorrhagic shock through the reduction of gut permeability. ${ }^{72}$ These studies suggest that vagus nerve activity is crucial for normal lung function, and the possibility of using VNS to improve outcome in lung injury has great promise for further research. In addition, preventing gut-barrier interaction that contributes to visceral inflammation also deserves further study.

\section{Stroke and TBI}

Stroke and TBI are also causes widespread neural inflammation which VNS may be able to alleviate. In a study conducted by Bansal et al, the effect of VNS on TBI was evaluated by measuring tissue and serum ghrelin and serum TNF $\alpha .^{75}$ Their study was based on the hypothesis that preventing the inflammatory surge after TBI could prevent sepsis, multiorgan failure, and other adverse effects. Since ghrelin is mediated through acetylcholine levels, it is reasonable to assume that VNS may have an application in treating TBI through a ghrelin or other hypothalamic-gated mechanism. ${ }^{75}$ VNS decreased the serum levels of TNF $\alpha$, an early cytokine marker for damage in trauma and ischemic injury. Regulation of cytokine expression by VNS may provide significant therapeutic value in these patients. Because VNS is known to have anti-inflammatory properties and affect the levels of acetylcholine, these changes in cytokine upregulation and rebalancing of neurotransmitter release may provide an immediate and controllable way to modulate injury due to stroke, ischemia, or trauma.

\section{Diabetes}

Diabetes is another inflammatory-related disorder that may benefit from treatment with VNS. Recent work has shown the role of the vagus nerve in the pathophysiology of diabetes and other related diseases, which may, in turn, suggest that VNS could be useful in treating such disorders. Cardiovascular risk has long been associated with diabetes, but the exact mechanism by which increased risk and diabetes synergize to exacerbate morbidity is not known. Pal et al found that relatives of type 2 diabetics had increased risk for cardiovascular diseases, which they attributed to sympathovagal imbalance. ${ }^{76}$ Changes in sympathovagal tone may underlie the increased autoinflammation that could be the foundation of this increased cardiovascular risk. Woie and Reed showed a relationship between changes in tracheal edema in control, diabetic, and insulin-treated diabetic rats which suggests that barrier breakdown was significantly greater in control animals but was attenuated in diabetic rats. ${ }^{77}$ Changes in airway secretion are vagally mediated, and the altered vagus tone in diabetes may exacerbate susceptibility to chronic inflammation. A broader concern is the role vagal tone plays in metabolic disease and obesity. Vagal afferents and projections to the hypothalamus play a significant role in satiety and feeding behavior, and disruption of vagal afferent traffic may contribute to obesity and downregulation of cholinergic descending tone to arrest inflammation. ${ }^{77}$ Meyers et al used selective efferent stimulation to significantly lower blood glucose, which may be a potential treatment for type 2 diabetes. ${ }^{78}$ While the interaction of inflammation and metabolic disorders is becoming clear, the potential role of VNS in treating diabetes needs further investigation. This study by Meyers et al uses a crude method of selective efferent stimulation, by cutting the vagus nerve above the stimulating electrode. However, selective blockade of vagal fibers using different electrical stimulation parameters may provide the answer for using VNS to affect metabolism. Since cutting the vagus nerve is not an ideal solution for human patients, similar results may be obtained by using different parameters that can selectively stimulate fiber types or afferent/efferent traffic. Patel and Butera have achieved such results by using high-frequency stimulation in both the vagus and the sciatic nerves in rats. ${ }^{54,79}$ Whether parameters 
for selective stimulation such as used by Patel et al could be used to replicate Meyers et al's results without the need to sever the vagus nerve remains to be determined.

\section{RA}

RA is a chronic, inflammatory autoimmune disease of unknown origin, which results in chronic synovial inflammation and damage to cartilage and bone due to release of cytokines and progressive inflammatory damage. ${ }^{80,81}$ Alpha

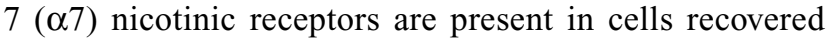
from synovial fluid and from synovial tissue, particularly in cells with a macrophage-like morphology. ${ }^{82}$ The general suppression of anti-inflammatory cholinergic pathways plays a critical role in RA. Das' review summarizes the current literature on the role that vagus tone plays in modulating RA and, as in so many of these inflammatory disorders, the preliminary evidence justifies further experiments. ${ }^{83}$ In a recently completed study by SetPoint Medical Corporation, VNS devices were implanted in patients with RA to test the safety and efficacy of treatment. Patients were assessed after 6 weeks of treatment and showed a $20 \%$ improvement in symptoms. ${ }^{84}$ Further experiments are needed to determine the role that VNS can play in the treatment of RA, in particular, the potential role of VNS in the modulation of cytokine cascade through $\alpha 7$ receptors.

\section{Conclusion}

VNS has been proven to be a useful treatment across a number of domains and has been used effectively to treat epilepsy and depression in adults. There is accumulating evidence to suggest that it can be used to help quell inflammation in a number of other autonomic or inflammatory disorders, which would make it useful for a wider range of pediatric patients as well. Preliminary studies have shown promise for VNS being used for stroke, autoimmune diseases, heart and lung failure, obesity, and pain management, but further studies are needed to fully elucidate the mechanistic actions that explain VNS's potential role in treating these disorders. Many of these studies are not mechanistic in nature, and further pathway analysis and studies focused on the mechanisms by which VNS alters autonomic tone are key to further our understanding of vagus nerve modification. VNS interacts with the body's immune system to modify inflammatory tone by altering the release of pro- and anti-inflammatory cytokines. We have summarized some of these key inflammatory markers in Figure 3. There is an overwhelming evidence to suggest that vagus nerve is an important component of the immune response and manipulating vagal tone is a way to modulate the immune

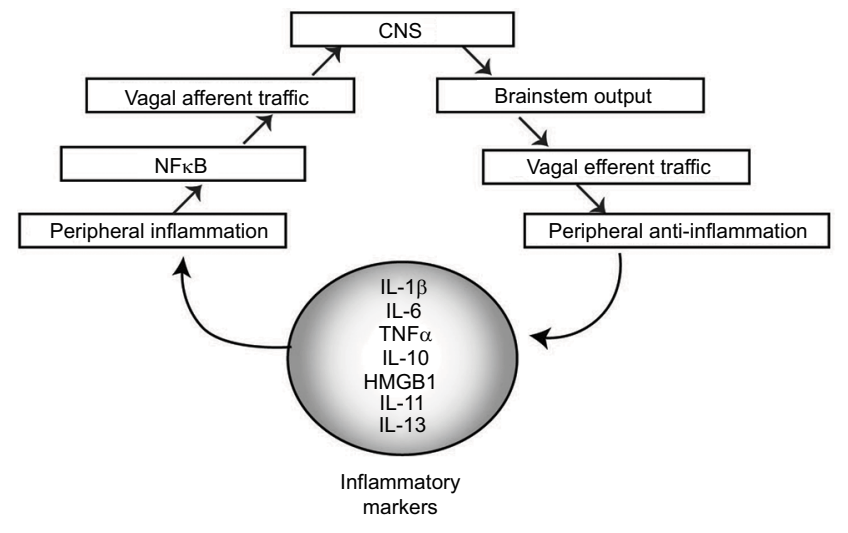

Figure 3 Flow diagram showing the inflammatory neural circuit.

Notes: Inflammatory markers including IL-I $\beta$, IL-6, TNF $\alpha$, IL-I0, HMGBI, IL-I I, and IL-13 alter peripheral inflammatory tone, which stimulates $\mathrm{NF \kappa B}$, resulting in increased inflammatory signaling to the CNS via the brain stem. This results in output that generates an inflammatory signal. VNS can be used to alter the input/ output of this autonomic control circuitry.

Abbreviations: IL, interleukin; TNF, tumor necrosis factor; NFKB, nuclear factor $\kappa \mathrm{B}$; CNS, central nervous system; VNS, vagus nerve stimulation.

system. Using VNS to manipulate vagal tone provides an exciting new opportunity for minimally invasive therapeutic intervention in adult and pediatric patients.

\section{Acknowledgment}

Funding for this work was provided by intramural funding through the GRASP and Pediatric Research Initiatives (Loma Linda University) to CGW.

\section{Disclosure}

The authors report no conflicts of interest in this work.

\section{References}

1. Bonaz B, Picq C, Sinniger V, Mayol JF, Clarençon D. Vagus nerve stimulation: from epilepsy to the cholinergic anti-inflammatory pathway. Neurogastroenterol Motil. 2013;25(3):208-221.

2. Tracey KJ. The inflammatory reflex. Nature. 2002;420(6917):853-859.

3. Borovikova LV, Ivanova S, Zhang M, et al. Vagus nerve stimulation attenuates the systemic inflammatory response to endotoxin. Nature. 2000; 405(6785):458-462.

4. Matteoli G, Gomez-Pinilla PJ, Nemethova A, et al. A distinct vagal anti-inflammatory pathway modulates intestinal muscularis resident macrophages independent of the spleen. Gut. 2014;63(6):938-948.

5. Rosas-Ballina M, Olofsson PS, Ochani M, et al. Acetylcholinesynthesizing T cells relay neural signals in a vagus nerve circuit. Science. 2011;334(6052):98-101.

6. Rosas-Ballina M, Ochani M, Parrish WR, et al. Splenic nerve is required for cholinergic antiinflammatory pathway control of TNF in endotoxemia. Proc Natl Acad Sci U S A. 2008;105(31):11008-11013.

7. Berthoud HR, Neuhuber WL. Functional and chemical anatomy of the afferent vagal system. Auton Neurosci. 2000;85(1-3):1-17.

8. Yu ZJ, Weller RA, Sandidge K, Weller EB. Vagus nerve stimulation: can it be used in adolescents or children with treatment-resistant depression? Curr Psychiatry Rep. 2008;10(2):116-122.

9. Browning KN, Travagli RA. Central nervous system control of gastrointestinal motility and secretion and modulation of gastrointestinal functions. Compr Physiol. 2014;4(4):1339-1368. 
10. Vivas AC, Baaj AA, Benbadis SR, Vale FL. The health care burden of patients with epilepsy in the United States: an analysis of a nationwide database over 15 years. Neurosurg Focus. 2012;32(3):E1.

11. Lanska DJ. J.L. Corning and vagal nerve stimulation for seizures in the 1880s. Neurology. 2002;58(3):452-459.

12. Couch JD, Gilman AM, Doyle WK. Long-term expectations of vagus nerve stimulation: a look at battery replacement and revision surgery. Neurosurgery. 2016;78(1):42-46.

13. Spuck S, Nowak G, Renneberg A, Tronnier V, Sperner J. Right-sided vagus nerve stimulation in humans: an effective therapy? Epilepsy Res. 2008;82(2-3):232-234.

14. Navas M, Navarrete EG, Pascual JM, et al. Treatment of refractory epilepsy in adult patients with right-sided vagus nerve stimulation. Epilepsy Res. 2010;90(1-2):1-7.

15. McGregor A, Wheless J, Baumgartner J, Bettis D. Right-sided vagus nerve stimulation as a treatment for refractory epilepsy in humans. Epilepsia. 2005;46(1):91-96.

16. Groves DA, Brown VJ. Vagal nerve stimulation: a review of its applications and potential mechanisms that mediate its clinical effects. Neurosci Biobehav Rev. 2005;29(3):493-500.

17. Manuals - Product Information / Manuals - Healthcare Professionals, on VNS Therapy for Epilepsy | Cyberonics US [webpage on the Internet]. Available from: http://us.livanova.cyberonics.com/en/vns-therapy-forepilepsy/healthcare-professionals/vns-therapy/manuals-page/. Accessed December 15, 2015.

18. Chakravarthy K, Chaudhry H, Williams K, Christo PJ. Review of the uses of vagal nerve stimulation in chronic pain management. Curr Pain Headache Rep. 2015;19(12):54.

19. Elliott RE, Rodgers SD, Bassani L, et al. Vagus nerve stimulation for children with treatment-resistant epilepsy: a consecutive series of 141 cases. J Neurosurg Pediatr. 2011;7(5):491-500.

20. Morris GL, Mueller WM. Long-term treatment with vagus nerve stimulation in patients with refractory epilepsy. The Vagus Nerve Stimulation Study Group E01-E05. Neurology. 1999;53(8):1731-1735.

21. Krahl SE, Clark KB. Vagus nerve stimulation for epilepsy: a review of central mechanisms. Surg Neurol Int. 2012;3(Suppl 4):S255-S259.

22. MacDonald SC, Bateman BT, McElrath TF, Hernández-Díaz S. Mortality and morbidity during delivery hospitalization among pregnant women with epilepsy in the United States. JAMA Neurol. 2015;72(9):981-988.

23. Patel SI, Pennell PB. Management of epilepsy during pregnancy: an update. Ther Adv Neurol Disord. 2016;9(2):118-129.

24. Houser MV, Hennessy MD, Howard BC. Vagal nerve stimulator use during pregnancy for treatment of refractory seizure disorder. Obstet Gynecol. 2010;115(2 Pt 2):417-419.

25. Salerno G, Passamonti C, Cecchi A, Zamponi N. Vagus nerve stimulation during pregnancy: an instructive case. Childs Nerv Syst. 2016;32(1):209-211.

26. Zack MM. National and state estimates of the numbers of adults and children with active epilepsy - United States, 2015. MMWR Morb Mortal Wkly Rep. 2017;66:821-825.

27. Cianchetti C, Messina P, Pupillo E, et al. The perceived burden of epilepsy: Impact on the quality of life of children and adolescents and their families. Seizure. 2015;24:93-101.

28. Terra VC, de Paola L, Silvado CE. Are children affected by epileptic neuropsychiatric comorbidities? Epilepsy Behav. 2014;38:8-12.

29. He W, Wang X-Y, Zhou L, et al. Transcutaneous auricular vagus nerve stimulation for pediatric epilepsy: study protocol for a randomized controlled trial. Trials. 2015;16:371.

30. Hallböök T, Lundgren J, Stjernqvist K, Blennow G, Strömblad L-G, Rosén I. Vagus nerve stimulation in 15 children with therapy resistant epilepsy; its impact on cognition, quality of life, behaviour and mood. Seizure. 2005;14(7):504-513.

31. Smyth MD, Tubbs RS, Bebin EM, Grabb PA, Blount JP. Complications of chronic vagus nerve stimulation for epilepsy in children. JNeurosurg. 2003;99(3):500-503.
32. Farwell JR, Lee YJ, Hirtz DG, Sulzbacher SI, Ellenberg JH, Nelson KB. Phenobarbital for febrile seizures - effects on intelligence and on seizure recurrence. $N$ Engl J Med. 1990;322(6):364-369.

33. Lagae L. Cognitive side effects of anti-epileptic drugs. The relevance in childhood epilepsy. Seizure. 2006;15(4):235-241.

34. Fernandez L, Gedela S, Tamber M, Sogawa Y. Vagus nerve stimulation in children less than 3 years with medically intractable epilepsy. Epilepsy Res. 2015;112:37-42.

35. Levy ML, Levy KM, Hoff D, et al. Vagus nerve stimulation therapy in patients with autism spectrum disorder and intractable epilepsy: results from the vagus nerve stimulation therapy patient outcome registry. J Neurosurg Pediatr. 2010;5(6):595-602.

36. Greenberg PE, Fournier A-A, Sisitsky T, Pike CT, Kessler RC. The economic burden of adults with major depressive disorder in the United States (2005 and 2010). J Clin Psychiatry. 2015;76(2):155-162.

37. Bajbouj M, Merkl A, Schlaepfer TE, et al. Two-year outcome of vagus nerve stimulation in treatment-resistant depression. JClin Psychopharmacol. 2010;30(3):273-281.

38. American Psychiatric Association. Diagnostic and Statistical Manual of Mental Disorders. Washington, DC: American Psychiatric Association; 1980.

39. Andrews G, Brugha T, Thase ME, Duffy FF, Rucci P, Slade T. Dimensionality and the category of major depressive episode. Int J Methods Psychiatr Res. 2007;16(Suppl 1):S41-S51.

40. Zimmerman M, Chelminski I, Posternak M. A review of studies of the Hamilton depression rating scale in healthy controls: implications for the definition of remission in treatment studies of depression. $J$ Nerv Ment Dis. 2004;192(9):595-601.

41. Nahas Z, Marangell LB, Husain MM, et al. Two-year outcome of vagus nerve stimulation (VNS) for treatment of major depressive episodes. J Clin Psychiatry. 2005;66(9):1097-1104.

42. Rush AJ, Kraemer HC, Sackeim HA, et al. Report by the ACNP Task Force on response and remission in major depressive disorder. Neuropsychopharmacology. 2006;31(9):1841-1853.

43. Weisskopf E, Fumeaux CJF, Bickle Graz M, et al. Bon usage des antidépresseurs ISRS durant la grossesse - le défi de l'évaluation de la balance bénéfice-risque. [SSRI antidepressant use during pregnancy and the assessment of the risk-benefit ratio]. Rev Médicale Suisse. 2016;12(510):561-566. French.

44. Preti A, Cardascia L, Zen T, et al. Obstetric complications in patients with depression - a population-based case-control study. JAffect Disord. 2000;61(1-2):101-106.

45. Husain MM, Stegman D, Trevino K. Pregnancy and delivery while receiving vagus nerve stimulation for the treatment of major depression: a case report. Ann Gen Psychiatry. 2005;4:16.

46. Judkins A, Johnson RL, Murray ST, Yellon SM, Wilson CG. Vagus nerve stimulation in pregnant rats and effects on inflammatory markers in the brain stem of neonates. Pediatr Res. 2018;83(2):514-519.

47. Botteron KN, Geller B. Refractory depression in children and adolescents. Depress Anxiety. 1997;5(4):212-223.

48. Antidepressant Medications for Children and Adolescents: Information for Parents and Caregivers. Available from: http://www.nimh.nih. gov/health/topics/child-and-adolescent-mental-health/antidepressantmedications-for-children-and-adolescents-information-for-parentsand-caregivers.shtml. Accessed January 6, 2016.

49. Slade E, Tamber PS, Vincent J-L. The Surviving Sepsis Campaign: raising awareness to reduce mortality. Crit Care Lond Engl. 2003; $7(1): 1-2$.

50. Kessler W, Diedrich S, Menges P, et al. The role of the vagus nerve: modulation of the inflammatory reaction in murine polymicrobial sepsis. Mediators Inflamm. 2012;2012:467620.

51. Huang J, Wang Y, Jiang D, Zhou J, Huang X. The sympathetic-vagal balance against endotoxemia. J Neural Transm (Vienna). 2010;117(6):729-735.

52. Johnson RL, Murray ST, Camacho DK, Wilson CG. Vagal nerve stimulation attenuates IL- 6 and TNF $\alpha$ expression in respiratory regions of the developing rat brain stem. Respir Physiol Neurobiol. 2016;229:1-4. 
53. Barbanti P, Grazzi L, Egeo G, Padovan AM, Liebler E, Bussone G. Noninvasive vagus nerve stimulation for acute treatment of high-frequency and chronic migraine: an open-label study. J Headache Pain. 2015;16:61.

54. Patel YA, Saxena T, Bellamkonda RV, Butera RJ. Kilohertz frequency nerve block enhances anti-inflammatory effects of vagus nerve stimulation. Sci Rep. 2017;7:39810.

55. Patel YA, Saxena T, Bellamkonda RV, Butera RJ. Corrigendum: kilohertz frequency nerve block enhances anti-inflammatory effects of vagus nerve stimulation. Sci Rep. 2017;7:46848.

56. Lange G, Janal MN, Maniker A, et al. Safety and efficacy of vagus nerve stimulation in fibromyalgia: a phase I/II proof of concept trial. Pain Med. 2011;12(9):1406-1413.

57. Randich A, Gebhart GF. Vagal afferent modulation of nociception. Brain Res Brain Res Rev. 1992;17(2):77-99.

58. Silberstein SD, Mechtler LL, Kudrow DB, et al. Non-invasive vagus nerve stimulation for the acute treatment of cluster headache: findings from the randomized, double-blind, sham-controlled ACT1 study. Headache. 2016;56(8):1317-1332.

59. FastStats [webpage on the Internet]. Obesity and overweight. Available from: http://www.cdc.gov/nchs/fastats/obesity-overweight.htm. Accessed January 7, 2016.

60. Burneo JG, Faught E, Knowlton R, Morawetz R, Kuzniecky R. Weight loss associated with vagus nerve stimulation. Neurology. 2002;59(3):463-464.

61. Bodenlos JS, Kose S, Borckardt JJ, et al. Vagus nerve stimulation acutely alters food craving in adults with depression. Appetite. 2007;48(2): 145-153.

62. Val-Laillet D, Biraben A, Randuineau G, Malbert CH. Chronic vagus nerve stimulation decreased weight gain, food consumption and sweet craving in adult obese minipigs. Appetite. 2010;55(2):245-252.

63. Raybould HE, Tache Y. Cholecystokinin inhibits gastric motility and emptying via a capsaicin-sensitive vagal pathway in rats. Am J Physiol. 1988;255(2):G242-G246.

64. Ikramuddin S, Blackstone RP, Brancatisano A, et al. Effect of reversible intermittent intra-abdominal vagal nerve blockade on morbid obesity: the ReCharge randomized clinical trial. JAMA. 2014;312(9):915-922.

65. Boron WF, Boulpaep EL. Medical Physiology, 2e Updated Edition: With STUDENT CONSULT Online Access. Atlanta, GA: Elsevier Health Sciences; 2012.

66. Das UN. Vagal nerve stimulation in prevention and management of coronary heart disease. World J Cardiol. 2011;3(4):105-110.

67. Sloan RP, McCreath H, Tracey KJ, Sidney S, Liu K, Seeman T. RR interval variability is inversely related to inflammatory markers: the CARDIA study. Mol Med Camb Mass. 2007;13(3-4):178-184.

68. Zhang Y, Popovic ZB, Bibevski S, et al. Chronic vagus nerve stimulation improves autonomic control and attenuates systemic inflammation and heart failure progression in a canine high-rate pacing model. Circ Heart Fail. 2009;2(6):692-699.

69. Zhao M, He X, Bi X-Y, Yu X-J, Gil Wier W, Zang W-J. Vagal stimulation triggers peripheral vascular protection through the cholinergic anti-inflammatory pathway in a rat model of myocardial ischemia/ reperfusion. Basic Res Cardiol. 2013;108(3):345.
70. Chapleau MW, Rotella DL, Reho JJ, Rahmouni K, Stauss HM. Chronic vagal nerve stimulation prevents high-salt diet-induced endothelial dysfunction and aortic stiffening in stroke-prone spontaneously hypertensive rats. Am J Physiol Heart Circ Physiol. 2016;311(1):H276-H285.

71. dos Santos CC, Shan Y, Akram A, Slutsky AS, Haitsma JJ. Neuroimmune regulation of ventilator-induced lung injury. Am J Respir Crit Care Med. 2011;183(4):471-482.

72. Levy G, Fishman JE, Xu D, et al. Vagal nerve stimulation modulates gut injury and lung permeability in trauma-hemorrhagic shock. J Trauma Acute Care Surg. 2012;73(2):338-342; discussion 342.

73. Tarras SL, Diebel LN, Liberati DM, Ginnebaugh K. Pharmacologic stimulation of the nicotinic anti-inflammatory pathway modulates gut and lung injury after hypoxia-reoxygenation injury. Surgery. 2013;154(4):841-847; discussion 847-848.

74. Reys LG, Ortiz-Pomales YT, Lopez N, et al. Uncovering the neuroenteric-pulmonary axis: vagal nerve stimulation prevents acute lung injury following hemorrhagic shock. Life Sci. 2013;92(13):783-792.

75. Bansal V, Ryu SY, Lopez N, et al. Vagal stimulation modulates inflammation through a ghrelin mediated mechanism in traumatic brain injury. Inflammation. 2012;35(1):214-220.

76. Pal GK, Adithan C, Ananthanarayanan PH, et al. Sympathovagal imbalance contributes to prehypertension status and cardiovascular risks attributed by insulin resistance, inflammation, dyslipidemia and oxidative stress in first degree relatives of type 2 diabetics. PLoS One. 2013;8(11):e78072.

77. Woie K, Reed RK. Alloxan diabetes abolishes the increased negativity of interstitial fluid pressure in rat trachea induced by vagal nerve stimulation. Acta Physiol Scand. 1997;161(1):113-119.

78. Meyers EE, Kronemberger A, Lira V, Rahmouni K, Stauss HM. Contrasting effects of afferent and efferent vagal nerve stimulation on insulin secretion and blood glucose regulation. Physiol Rep. 2016;4(4):e12718.

79. PatelYA, Butera RJ. Differential fiber-specific block of nerve conduction in mammalian peripheral nerves using kilohertz electrical stimulation. J Neurophysiol. 2015;113(10):3923-3929.

80. Feldmann M, Brennan FM, Maini RN. Role of cytokines in rheumatoid arthritis. Annu Rev Immunol. 1996;14:397-440.

81. Tetta C, Camussi G, Modena V, Di Vittorio C, Baglioni C. Tumour necrosis factor in serum and synovial fluid of patients with active and severe rheumatoid arthritis. Ann Rheum Dis. 1990;49(9):665-667.

82. Westman M, Engström M, Catrina AI, Lampa J. Cell specific synovial expression of nicotinic alpha 7 acetylcholine receptor in rheumatoid arthritis and psoriatic arthritis. Scand J Immunol. 2009;70(2):136-140.

83. Das UN. Can vagus nerve stimulation halt or ameliorate rheumatoid arthritis and lupus? Lipids Health Dis. 2011;10:19.

84. ClinicalTrials.gov [webpage on the Internet]. Safety and Efficacy Vagal Nerve Stimulation in Patients with Rheumatoid Arthritis - Tabular View; 2012. Available from: https://clinicaltrials.gov/ct2/show/NCT0155294 1?term=NCT01552941\&rank=1. Accessed January 7, 2016.

85. Kirchberger K, Hummel C, Stefan H. Postoperative multichannel magnetoencephalography in patients with recurrent seizures after epilepsy surgery. Acta Neurol Scand. 1998;98(1):1-7.
Journal of Inflammation Research

\section{Publish your work in this journal}

The Journal of Inflammation Research is an international, peer-reviewed open access journal that welcomes laboratory and clinical findings on the molecular basis, cell biology and pharmacology of inflammation including original research, reviews, symposium reports, hypothesis formation and commentaries on: acute/chronic inflammation; mediators of inflammation; cellular processes; molecular mechanisms; pharmacology and novel anti-inflammatory drugs; clinical conditions involving inflammation. The manuscript management system is completely online and includes a very quick and fair peer-review system. Visit http://www.dove press.com/testimonials.php to read real quotes from published authors. 